\title{
Notch inhibition overcomes resistance to tyrosine kinase inhibitors in EGFR-driven lung adenocarcinoma
}

\begin{abstract}
Emilie Bousquet Mur, ${ }^{1}$ Sara Bernardo, ${ }^{1}$ Laura Papon, ${ }^{1}$ Maicol Mancini, ${ }^{1}$ Eric Fabbrizio,, ${ }^{1}$ Marion Goussard, ${ }^{1}$ Irene Ferrer, ${ }^{1,2,3}$ Anais Giry, ${ }^{1}$ Xavier Quantin, ${ }^{1}$ Jean-Louis Pujol, ${ }^{1,4}$ Olivier Calvayrac, ${ }^{5}$ Herwig P. Moll, ${ }^{6}$ Yaël Glasson, ${ }^{7}$ Nelly Pirot, ${ }^{7}$ Andrei Turtoi, ${ }^{8}$ Marta Cañamero, ${ }^{9}$ Kwok-Kin Wong, ${ }^{10}$ Yosef Yarden,, ${ }^{11}$ Emilio Casanova, ${ }^{6,12}$ Jean-Charles Soria, ${ }^{13}$ Jacques Colinge, ${ }^{14}$ Christian W. Siebel, ${ }^{15}$ Julien Mazieres, ${ }^{5,16}$ Gilles Favre, ${ }^{5}$ Luis Paz-Ares, ${ }^{2,4,17}$ and Antonio Maraver ${ }^{1}$

IInstitut de Recherche en Cancérologie de Montpellier (IRCM), Université de Montpellier, Institut Régional du Cancer de Montpellier (ICM), Montpellier, France. Unidad de Investigación Clínica de Cáncer de Pulmón, Instituto de Investigación Hospital 12 de Octubre-CNIO, Madrid, Spain. ${ }^{3}$ CIBERONC, Madrid, Spain. ${ }^{4}$ Montpellier Academic Hospital, Hôpital Arnaud de Villeneuve, Montpellier, France. ${ }^{5}$ INSERM, Centre de Recherche en Cancérologie de Toulouse, CRCT UMR-1037, Toulouse, France; Institut Claudius Regaud, IUCT-Oncopole, Laboratoire de Biologie Médicale Oncologique, Toulouse, France; University of Toulouse III (Paul Sabatier), Toulouse, France. ${ }^{6}$ Department of Physiology, Center of Physiology and Pharmacology and Comprehensive Cancer Center (CCC), Medical University of Vienna, Vienna, Austria. ${ }^{7}$ Réseau d'Histologie Expérimentale de Montpellier, BioCampus, UMS3426 CNRS-USO09 INSERM-UM, Montpellier, France. ${ }^{8}$ IRCM, Université de Montpellier, ICM, Montpellier, France. ${ }^{9}$ Roche Pharmaceutical Research and Early Development, Translational Medicine, Roche Innovation Center, Munich, Germany. ${ }^{10}$ Laura and Isaac Perlmutter Cancer Center, NYU Langone Medical Center, New York, New York, USA ${ }^{11}$ Department of Biological Regulation, Weizmann Institute of Science, Rehovot, Israel. ${ }^{12}$ Ludwig Boltzmann Institute for Cancer Research (LBI-CR), Vienna, Austria. ${ }^{13}$ Drug Development Department (DITEP) Gustave Roussy Cancer Campus, Paris-Sud University, Villejuif, France. ${ }^{14}$ IRCM, Université de Montpellier, ICM, Montpellier, France. ${ }^{15}$ Department of Discovery Oncology, Genentech, Inc., South San Francisco, California, USA. ${ }^{16}$ Thoracic Oncology Department, Larrey Hospital, University Hospital of Toulouse, France; INSERM, Centre de Recherche en Cancérologie de Toulouse, CRCT UMR-1037, Toulouse, France; University of Toulouse III (Paul Sabatier), Toulouse, France. ${ }^{17}$ Medical School, Universidad Complutense, Madrid, Spain.
\end{abstract}

EGFR-mutated lung adenocarcinoma patients treated with gefitinib and osimertinib show a therapeutic benefit limited

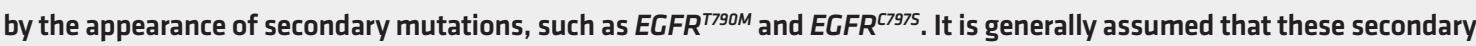
mutations render ECFR completely unresponsive to the inhibitors, but contrary to this, we uncovered here that gefitinib and osimertinib increased STAT3 phosphorylation (p-STAT3) in EGFR ${ }^{\text {T790M }}$ and EGFR ${ }^{\text {C7975 }}$ tumoral cells. Interestingly, we also found that concomitant Notch inhibition with gefitinib or osimertinib treatment induced a p-STAT3-dependent strong reduction in the levels of the transcriptional repressor HES1. Importantly, we showed that tyrosine kinase inhibitor-resistant tumors, with $E C F R^{T 790 M}$ and ECFR ${ }^{C 7975}$ mutations, were highly responsive to the combined treatment of Notch inhibitors with gefitinib or osimertinib, respectively. Finally, in patients with EGFR mutations treated with tyrosine kinase inhibitors, HES1 protein levels increased during relapse and correlated with shorter progression-free survival. Therefore, our results offer a proof of concept for an alternative treatment to chemotherapy in lung adenocarcinoma osimertinib-treated patients after disease progression.

\section{Introduction}

Lung cancer kills approximately 1 million people every year worldwide and is the leading cause of death by cancer in the world. Lung cancer consists of 2 main types: small cell lung carcinoma that accounts for about $20 \%$ of lung cancers and non-small cell lung carcinoma, divided into lung adenocarcinoma, lung squamous carcinoma, and large cell carcinoma, that account for approximately $40 \%, 30 \%$, and $10 \%$ of all lung cancer, respectively (1). Genetic alterations in the EGFR gene is encountered in about $20 \%$ of lung adenocarcinoma patients in Western countries, and up to $50 \%$ in some Asian countries such as Korea. The most common ones are deletions in exon 19 and the activating EGFR ${ }^{L 858 R}$ mutation (2). The life expectancy of this subset of patients has improved dramatically thanks to the development of tyrosine kinase inhibitors (TKIs) (3). Most of the patients treated with first-generation

Conflict of interest: The authors have declared that no conflict of interest exists. Copyright: @ 2019, American Society for Clinical Investigation.

Submitted: December 18, 2018; Accepted: October 15, 2019; Published: December 17, 2019 Reference information: / Clin Invest. 2020;130(2):612-624.

https://doi.org/10.1172/JCl126896.
TKIs (i.e., gefitinib and erlotinib) initially respond well; however, their tumors rapidly develop resistance. This is explained, in about $60 \%$ of cases, by acquisition of the so-called gatekeeper mutation $E G F R^{T 790 M}$ (4). More recently, third-generation TKIs, such as osimertinib, targeting $E G F R^{T 790 M}$ showed very good therapeutic response in patients expressing this mutation (5). Unfortunately, tumors from patients treated with osimertinib also become resistant to this drug; in around $30 \%$ of cases this is due to acquisition of new gatekeeper mutations, such as $\operatorname{EGFR}^{\mathrm{C} 7975}(6,7)$. Thus, a single drug to efficiently treat EGFR-driven lung adenocarcinoma might have limited value and a strategy based on combinational drug therapy could be more effective at mitigating the effects of gatekeeper mutations.

The resistance conferred by the EGFR ${ }^{T 790 M}$ gatekeeper mutation is multifactorial, including drug binding that is weakened through steric hindrance as well as an increase in the affinity for ATP in EGFR (8). Still, the binding of gefitinib in the presence of the $E G F R^{T 790}$ gatekeeper mutation, although negatively affected, is not totally inhibited (8). Moreover, $\mathrm{x}$-ray crystal structure analysis indicates that gefitinib binds to EGFR in a similar manner in the presence or absence of the $E G F R^{T 790 M}$ gatekeeper mutation (9). 
Hence, we hypothesized that although not achieving a therapeutic effect, gefitinib could to a certain extent impact EGFR downstream signaling pathways and this could be exploited upon combined inhibition of other signaling pathways.

The Notch signaling pathway is highly conserved among metazoans and it is important during embryonic development as well as adult tissue homeostasis. In mammals, there are $4 \mathrm{NOTCH}$ receptors (NOTCH1 to -4 ), that are activated upon interaction with transmembrane ligands (DELTA and JAGGED). For this activation to occur, an intramembrane protease called $\gamma$-secretase releases the Notch intracytoplasmic domain (NICD) that, upon nuclear translocation and binding to its DNA binding partner RBPJ, modulates the expression of target genes of the canonical Notch pathway, such as HES1 (10). The Notch pathway may thus be inhibited by $\gamma$-secretase inhibitors (GSIs) or by antibodies against the ligands or the receptors (11).

By making use of genetically engineered mouse models, we and others have demonstrated that KRAS-driven lung adenocarcinoma is dependent on Notch activity (12-14). Regarding EGFR-driven lung adenocarcinoma, seminal work using cell lines and murine subcutaneous xenografts showed that a combination of Notch inhibitors and EGFR TKIs produces a better response than single treatments in sensitive cells (15-17). However, the mechanism underlying this positive effect is not fully understood, and moreover, the role of the Notch pathway in lung adenocarcinoma that relapsed due to acquisition of gatekeeper mutations in EGFR remains largely unknown.

In this study, several pathways, including the KRAS signaling pathway, were downregulated in transcriptomic analysis performed upon treatment with gefitinib in EGFR-driven lung adenocarcinoma of human cells harboring the $E G F R^{T 790 M}$ gatekeeper mutation. Hence, based on our previous work (14), we combined TKIs with Notch inhibition in the presence of EGFR gatekeeper mutations and, importantly, found that this approach in vivo resensitizes human and murine lung adenocarcinoma resistant to gefitinib via phosphorylated STAT3 (p-STAT3) binding to the HES1 promoter, thus repressing HES1 expression. Similarly, Notch inhibition in vivo resensitizes human lung adenocarcinoma cells harboring the EGFR ${ }^{C 797 S}$ mutation to osimertinib, which most probably will soon become the first line of treatment in EGFR-driven lung adenocarcinoma patients. Altogether, our data show that Notch inhibition could be a potent strategy to treat TKI-resistant EGFR-driven lung adenocarcinoma patients.

\section{Results}

Gefitinib treatment in human lung adenocarcinoma cells with the gatekeeper mutation EGFR ${ }^{T 790 M}$ induces changes in several cancerassociated genetic signatures. To identify molecular changes upon gefitinib treatment in lung cancer cells harboring the $E G F R^{T 790 M}$ mutation that confers resistance to first-generation TKIs, we used the previously described human EGFR-driven lung adenocarcinoma PC9GR cell line $\left(E G F R^{T 790 M}\right)$ that is resistant to gefitinib (18). Gene set enrichment analysis using the Molecular Signatures Database Hallmark Gene Set Collection $(19,20)$ of data obtained by RNA sequencing (RNA-Seq) of PC9GR cells treated with vehicle or gefitinib showed that among the 50 signatures, only 1 was upregulated (HALLMARK_KRAS_SIGNALING_DN) (Supplemen- tal Table 1; supplemental material available online with this article; https://doi.org/10.1172/JCI126896DS1). Accordingly, among the 8 downregulated gene sets in gefitinib-treated cells, we found HALLMARK_KRAS_SIGNALING_UP (Figure 1A and Supplemental Table 1). This suggests that in PC9GR cells, gefitinib decreases the activity of the KRAS signaling pathway, a well-known EGFR downstream pathway (21).

We previously reported that the Notch pathway plays a major role in KRAS-driven lung adenocarcinoma, and that its inhibition fully stops tumor growth in this setting (14). Therefore, we hypothesized that gefitinib's effects in PC9GR cells harboring the EGFR ${ }^{T 790 M}$ gatekeeper mutation could be enhanced by Notch inhibition.

Inhibition of Notch signaling hampers tumor growth in $E G F R^{T 790 M / L 858 R}$ mice. Before directly testing this hypothesis, we studied the Notch pathway activation in EGFR-driven lung tumors in vivo, by crossing $E G F R^{T 790 M / L 858 R}$ (22) and lung-specific CCSP-rtTA-transgenic mice (23) to obtain mice in which $E G F R^{T 790 M / L 858 R}$ expression in lungs can be induced by treatment with doxycycline ( $E G F R^{T 790 M / L 858 R}$ mice, hereafter). After 8 weeks of doxycycline treatment, mice developed bronchial and peripheral $E G F R^{T 790 M / L 858 R}$-driven tumors that are resistant to first-generation EGFR TKIs, such as gefitinib (22). Western blot analysis showed that N1ICD, the processed and active form of NOTCH1, and HES1, a Notch target gene, were strongly expressed in $E G F R^{T 790 M / L 858 R}$-driven tumors compared with normal lung tissue from control mice (either littermates with the same genotype but not treated with doxycycline, or CCSP-rtTA-transgenic mice treated with doxycycline) (Figure 1B). This finding is similar to what was observed in the Kras ${ }^{G 12 V}$ mouse model (14), and suggests that the Notch pathway may play a similar role in both tumor types.

Because the NOTCH1 and NOTCH3 receptors promote $\mathrm{Kras}^{\mathrm{G}}{ }^{12 \mathrm{~V}}$-driven lung adenocarcinoma, whereas NOTCH2 has a tumor suppressive role $(12,13,24)$, we analyzed their expression in $E G F R^{T 790 M / L 858 R}$-driven lung adenocarcinoma. The transmembrane forms of NOTCH1 and NOTCH3 (i.e., before cleavage by $\gamma$-secretase) were strongly expressed in tumor samples compared with controls (Figure 1B), whereas NOTCH2 expression was comparable in both groups (Figure 1B). Although the level of the transmembrane forms of NOTCH receptors does not reflect Notch activity, and NOTCH3 can be a direct target of NOTCH1 in some circumstances, this finding suggests that both NOTCH1 and NOTCH3 are mediators of the Notch pathway in EGFRdriven tumors in vivo.

To test whether Notch pathway activity is necessary for the growth of EGFR-driven tumors, we treated $E G F R^{T 790 M / L 858 R}$ mice with doxycycline for 8 weeks to induce tumor formation, and then randomly assigned them to 3 groups: (i) control group, treated with vehicle and IgG antibody control; (ii) GSI group, treated with dibenzazepine (DBZ), a potent and selective GSI; and (iii) anti-NRR1/NRR3 group, treated with blocking antibodies against NOTCH1 and NOTCH3, according to previously described treatment regimens (25-27). After 5 weeks of treatment, tumors represented more than $40 \%$ of the lung area in the control group, but only $20 \%$ and $10 \%$ in the DBZ and anti-NRR1/NRR3 groups, respectively (Figure 1C). This indicates that the Notch pathway is required for $E G F R^{T 790 M / L 858 R}$-driven tumor growth. Body weight was comparable in the 3 groups (Supplemental Figure 1A), sug- 
A

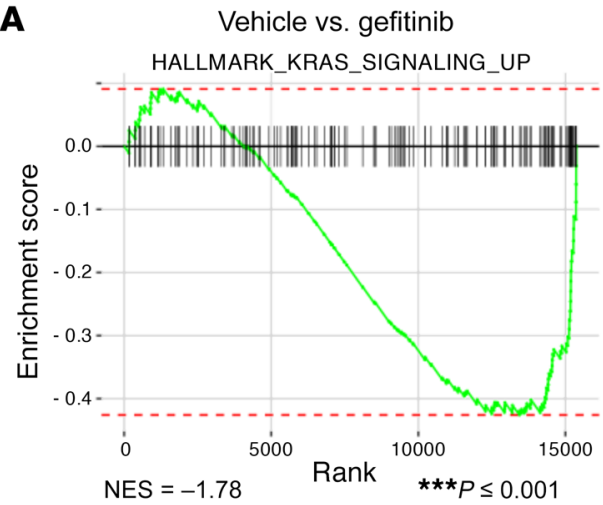

B

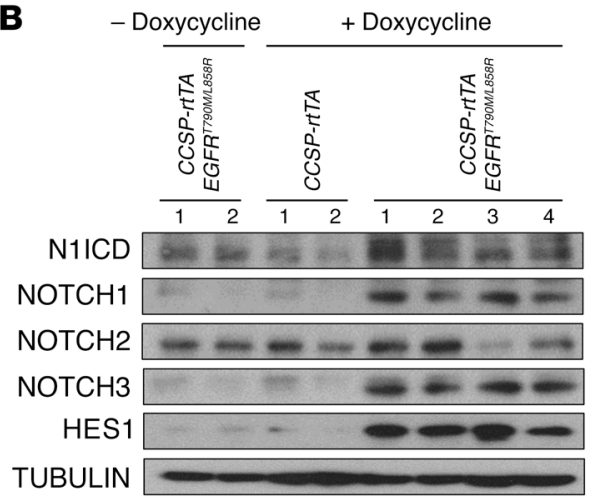

C

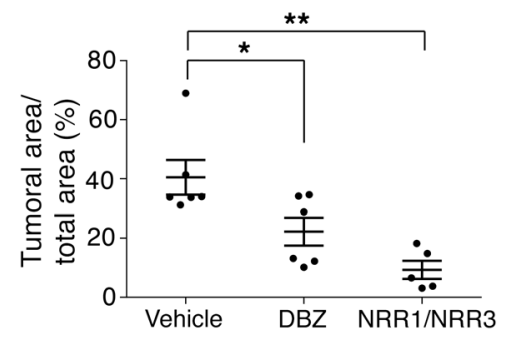

D

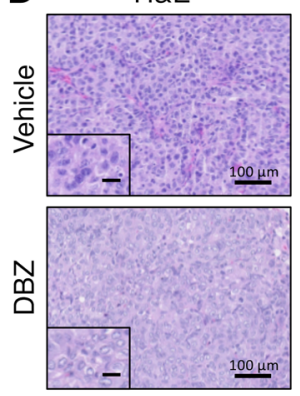

HES1

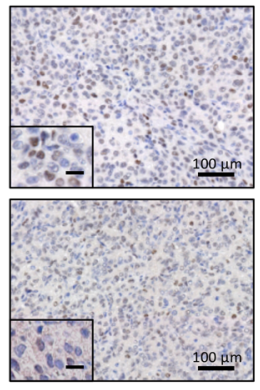

Ki67

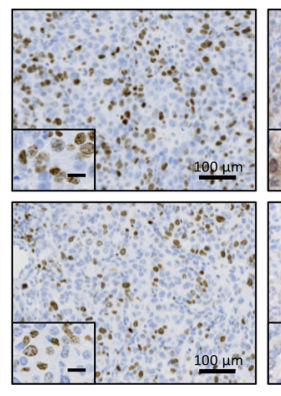

p-ERK

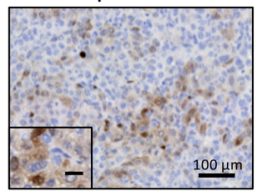

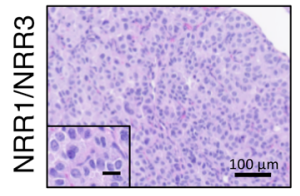

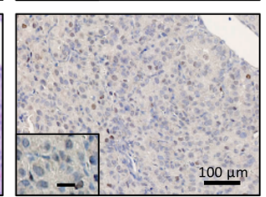

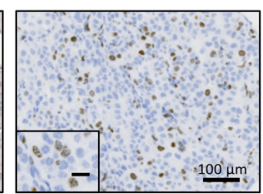
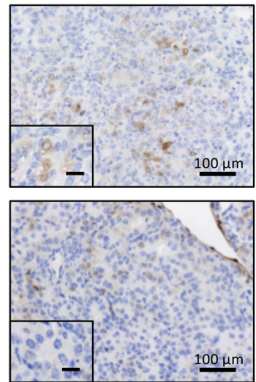
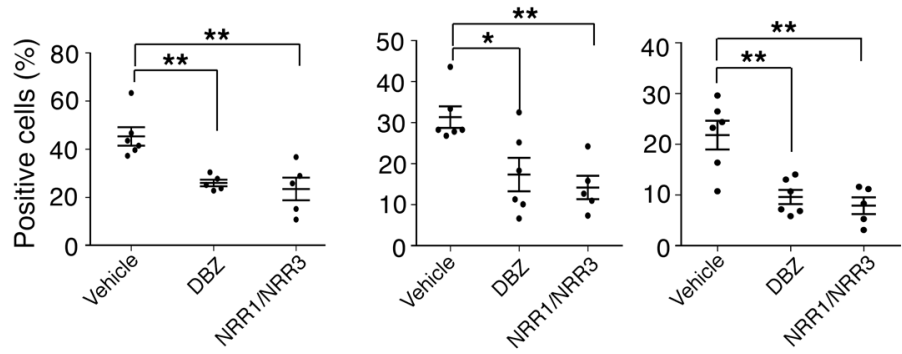

Figure 1. Inhibition of Notch signaling hampers tumor growth in EGFR ${ }^{\text {T790M/L858R }}$ mice. (A) PC9GR cells were starved for 18 hours and then treated for 6 hours with vehicle (DMSO) or gefitinib $(1 \mu \mathrm{M})$. RNA was extracted from cells and subjected to RNA-Seq. The KRAS-associated gene set was downregulated in PC9GR cells treated with gefitinib ( $n=3$ per genotype; FDR $<0.001$ ). NES, normalized enrichment score. (B) Immunoblotting of the indicated proteins in lungs from control mice and in EGFR ${ }^{T 790 M / L 858 R}$-driven tumors $(n=4)$. The controls were littermates of EGFR ${ }^{T 790 M / L 858 R}$ mice that were not induced with doxycycline $(n=2)$ or CCSP-rtTA mice treated with doxycycline $(n=2)$. (C) Tumor area as a percentage of total lung area of mice treated with methocel and IgC (vehicle; $n=6$ ), with $\gamma$-secretase inhibitor (DBZ; $n=6$ ), or with anti-NOTCH1 and anti-NOTCH3 antibodies (NRR1/NRR3; $n=5$ ) was determined by staining tissue sections with $\mathrm{H} \& \mathrm{E}$. (D) H\&E and immunohistochemical staining of lung tumors from the same mice as in $\mathbf{C}$. The dot plots show the percentage of positive cells in the corresponding immunohistochemically stained sections. They correspond to the analysis of 5 fields (original magnification, $\times 10$ ) per tumor. Scale bars: $100 \mu \mathrm{m}$; Inset scale bars: $25 \mu \mathrm{m}$. Values correspond to the average \pm SEM. Statistical significance in $\mathbf{C}$ and $\mathbf{D}$ was determined by 1 -way ANOVA followed by Tukey's post hoc test. ${ }^{*} P \leq 0.05,{ }^{* *} P \leq 0.01$.

gesting the absence of the intestinal toxicity reported by other studies using regimens that led to stronger Notch inhibition (28).

As expected, analysis of protein expression by immunohistochemistry (IHC) of tumors from anti-NRR1/NRR3- or DBZ-treated mice showed fewer HES1-positive cells than in the vehicle-treated control group, implying that these treatments effectively inhibited the Notch pathway (Figure 1D). Moreover, the percentage of Ki67-positive cells was lower in tumors from the anti-NRR1/NRR3 and DBZ groups than the control group, indicating that Notch activ- ity promotes cell proliferation in $E G F R^{T 790 M / L 858 R}$-driven tumors (Figure 1D). Because the MAPK and AKT pathways are crucial downstream players of the EGFR signaling pathway (21), we also analyzed the expression of p-ERK and p-AKT in the same samples. The percentage of p-ERK-positive cells was similarly reduced by treatment with the anti-NRR1 and -NRR3 antibodies and with DBZ compared with control (Figure 1D), consistent with previous observations $(13,14)$. Conversely, the percentage of p-AKT-positive cells was comparable in all groups (Supplemental Figure 1B). 
Notch inhibition overcomes resistance to gefitinib in $E_{\text {GFR }}{ }^{T 790 M / L 858 R_{-}}$ driven lung adenocarcinoma. To study whether pharmacological inhibition of the Notch pathway in vivo had any impact on the resistance to gefitinib conferred by the gatekeeper mutation $E G F R^{T 790 M}$, we randomized $E G F R^{T 790 M / L 858 R}$ mice (after 8 weeks of doxycycline treatment) in 4 treatment groups: (i) vehicle (control), (ii) gefitinib, (iii) DBZ, and (iv) gefitinib plus DBZ. For simplicity we decided to inhibit the Notch pathway hereafter only with a GSI.

As before, body weight was comparable in the different groups after the 5 weeks of treatment, suggesting that these drugs were well tolerated alone or in combination (Supplemental Figure 2A). In agreement with the previous findings (Figure 1C), tumor tissue occupied $42 \%$ of the lung in the control group, whereas it was decreased to $23 \%$ in the DBZ group (Figure 2A). As expected, gefitinib alone did not have any antitumor effect in $E G F R^{T 790 M / L 858 R}$ mice (52\% of lung was tumor tissue). Conversely, the DBZ plus gefitinib combination led to a very significant reduction of the tumor area compared with DBZ alone (tumor tissue covered only $10 \%$ of the total lung area) (Figure 2A).

Histopathological analysis of lung adenocarcinoma samples (i.e., nonbenign tumors, Supplemental Figure 2B) showed that the single treatments had no effect on the lung adenocarcinoma number compared with control (i.e., vehicle-treated mice) (Figure 2B). Importantly, animals treated with the combination of gefitinib and Notch inhibition had significantly fewer lung adenocarcinomas than vehicle-treated ones (a mean of 10 lung adenocarcinoma per mouse vs. 31 in the control, Figure 2B).

IHC analysis showed that the percentage of HES1-, Ki67-, p-ERK-, and p-AKT-positive cells was comparable in tumors from the gefitinib group and from controls (Figure 2C). By contrast, the percentage of HES1-, Ki67-, and p-ERK-positive cells was reduced in tumors from DBZ-treated mice (Figure 2C), as before (Figure 1D), although in this case the difference was not significant for p-ERK. The percentage of HES1-, Ki67-, and p-ERK-positive cells tended to be lower in mice treated with the gefitinib and DBZ combination compared with DBZ-treated mice, particularly for p-ERK. Finally, the percentage of p-AKT-positive cells was comparable in the DBZ, gefitinib, and control groups, but interestingly, it was significantly reduced in the gefitinib plus DBZ group compared with control mice (Figure 2C).

Altogether, these data demonstrate that inhibition of Notch signaling by DBZ restores sensitivity to treatment with gefitinib in $E G F R^{T 790 M / L 858 R}$-driven lung adenocarcinoma in vivo.

Notch inhibition overcomes resistance to gefitinib in lung adenocarcinoma patient-derived xenografts with EGFR ${ }^{T 790 M / L 858 R}$ mutations. These results were very encouraging; however, it is considered that the best strategy for testing innovative cancer treatments is the combination of genetic mouse models and patient-derived xenograft (PDX) preclinical models (29). Therefore, we developed a lung adenocarcinoma PDX that harbors the $E G F R^{T 790 M / L 858 R}$ mutations, like our transgenic mouse model. One week after subcutaneous grafting of the PDX, nude mice were randomized in 4 groups as before: (i) vehicle alone (control), (ii) gefitinib, (iii) DBZ, and (iv) gefitinib plus DBZ. Tumor growth was monitored for 30 days (i.e., the treatment duration). As expected, the $E G F R^{T 790 M}$ mutation conferred resistance to gefitinib. On the other hand, DBZ inhibited tumor growth, and strik- ingly, the DBZ and gefitinib combination almost totally blocked tumor growth (Figure 3A).

As before, IHC analysis of tumors showed that DBZ (alone or in combination with gefitinib) efficiently decreased the percentage of HES1-positive cells compared with control (Figure 3B). Tumor cell proliferation (Ki67-positive cells) was reduced by DBZ alone, and this effect was increased by addition of gefitinib. Similarly, the percentage of p-ERK-positive cells was decreased by treatment with DBZ alone and even more by the DBZ and gefitinib combination compared with control. This indicated that the DBZ and gefitinib combination was more effective in reducing MAPK signaling than Notch inhibition alone. Finally, the percentage of p-AKT-positive cells also was efficiently and similarly reduced by DBZ and by the DBZ plus gefitinib combination.

Altogether, our results provide strong preclinical evidence for the likely therapeutic benefit of combining Notch inhibition and gefitinib in patients with TKI-resistant EGFR-driven lung adenocarcinoma harboring the gatekeeper mutation $E G F R^{T 790 M}$.

Combining EGFR TKIs and Notch inhibitors synergistically decreases HES1 expression. Our previous analysis showed that the DBZ and gefitinib combination is more efficient than each single treatment in reducing MAPK and AKT pathways. Previous reports, including work from our laboratory, identified HES1 as an important positive MAPK regulator in KRAS-driven lung adenocarcinoma $(13,30)$. Moreover, HES1 has a similar effect on AKT signaling in $\mathrm{T}$ cell acute lymphoblastic leukemia (T-ALL) (31). Therefore, we hypothesized that HES1 could be an important mediator of p-ERK and p-AKT upon treatment with the DBZ and gefitinib combination. As the percentage of HES1-positive cells was similar in tumors from mice treated with DBZ alone and the DBZ plus gefitinib combination in both preclinical models (Figure 2C and Figure 3B), we analyzed HES1 signal intensity in the same samples. Importantly, HES1 signal intensity was significantly lower in tumors from mice treated with the DBZ and gefitinib combination than from mice treated with DBZ alone in the PDX model, and followed a similar trend in $E G F R^{T 790 M / L 858 R}$ mice (Figure 4, A and B).

To further validate our data, we analyzed HES1 expression by Western blotting in PC9GR cells (previously used for the RNA-Seq analysis, Figure 1A and Supplemental Table 1) after incubation with the different drugs alone or in combination. In agreement with our in vivo observation, HES1 expression was strongly reduced in cells exposed to the DBZ and gefitinib combination (Figure 4C).

Then, to explore HES1's role in PC9GR cells, we depleted HES1 using a pool of siRNAs targeting HES1 mRNA (siHES1) (Supplemental Figure 3). Of note, proliferation of siHES1-treated cells was impaired compared with control cells transfected with the nontargeted siRNA $(s i N T)$, and this effect was potentiated in the presence of gefitinib (Figure 4D).

To test whether the gefitinib effect was mediated by EGFR, we used the Chinese hamster ovary $(\mathrm{CHO})$ cell line that is naturally null for EGFR and was previously used for EGFR gain-offunction analyses (32). Interestingly, HES1 expression was not affected by cotreatment with DBZ and gefitinib in CHO cells transfected with empty vector, but was reduced in $\mathrm{CHO}$ cells that express EGFR ${ }^{\mathrm{T} 790 \mathrm{M} / \mathrm{L} 858 \mathrm{R}}$ protein (Figure $4 \mathrm{E}$ ). We concluded that EGFR is needed for HES1 expression reduction by the DBZ and gefitinib combination. 
A

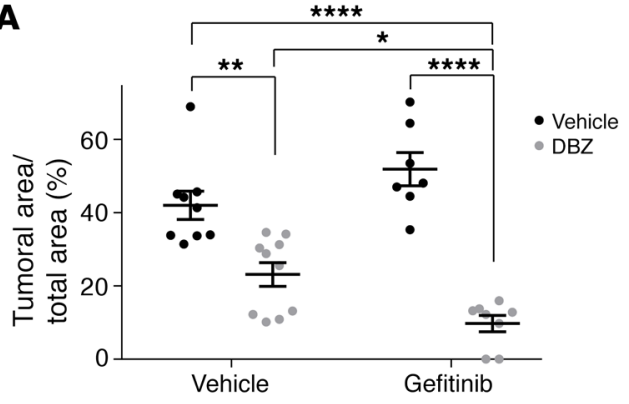

C
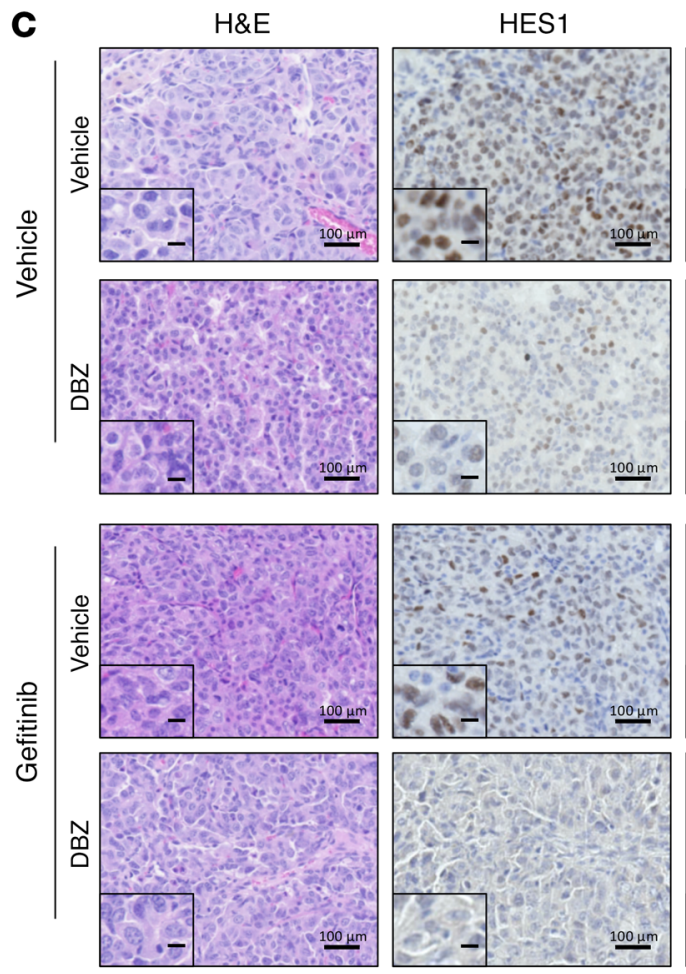

B

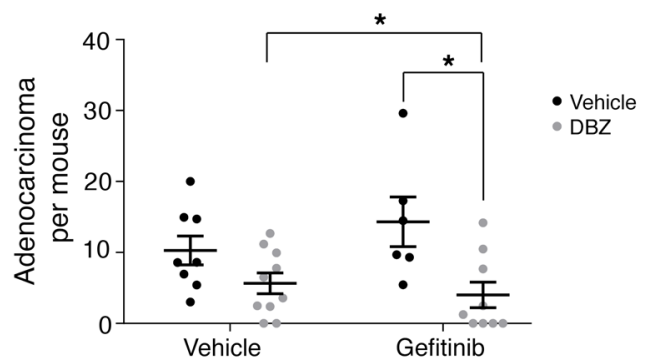

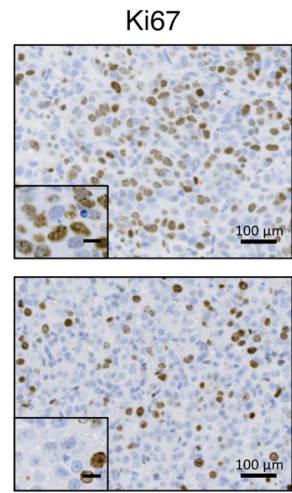
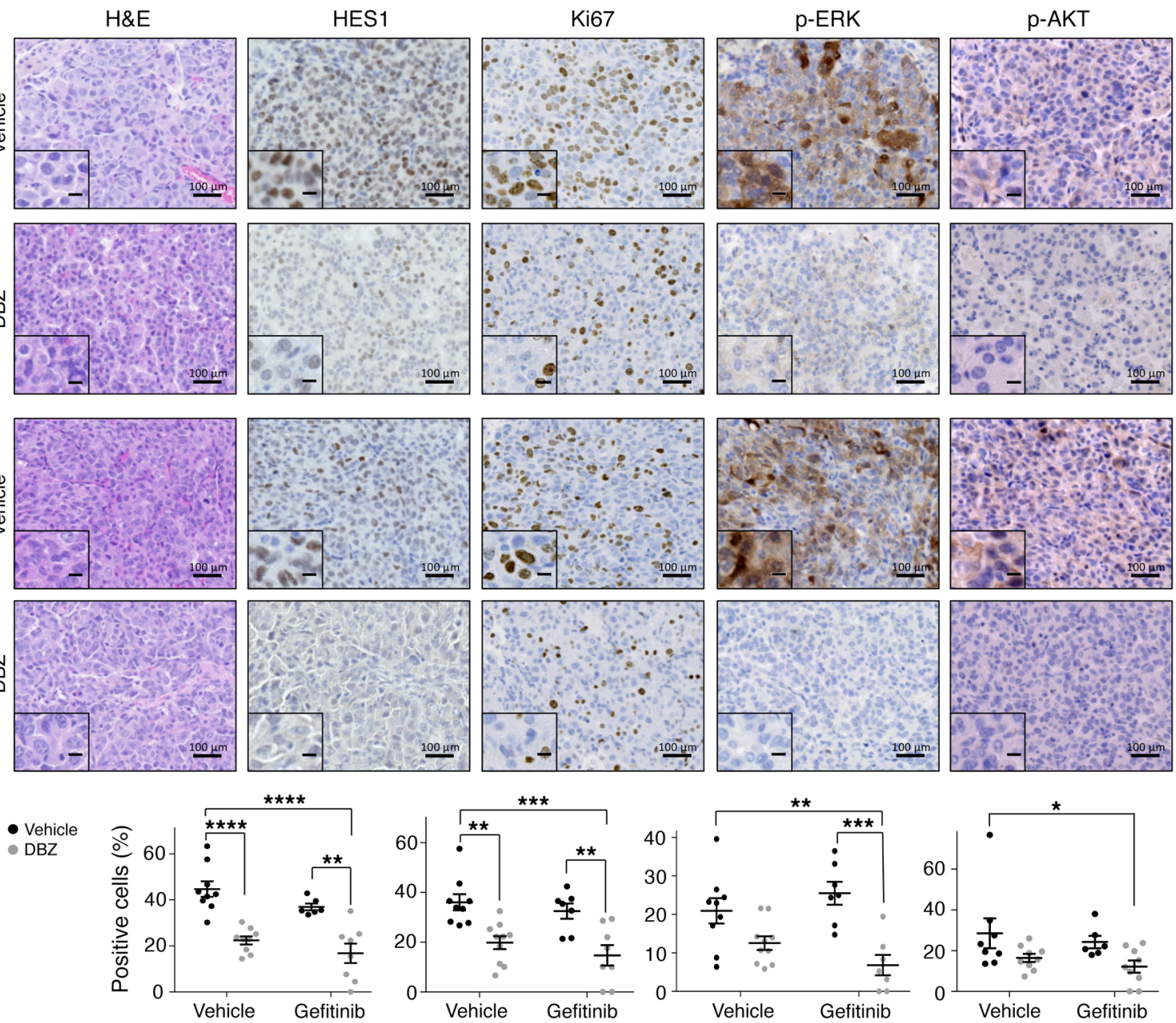

Figure 2. Notch inhibition sensitizes mouse EGFR ${ }^{\text {T90M/L858R }}$-driven tumors to gefitinib. (A) Tumor area as a percentage of the total lung area in sections of lung tissue from mice treated with methocel (vehicle; $n=9$ ), with $\gamma$-secretase inhibitor (DBZ; $n=10)$, with gefitinib $(n=7)$, or with a combination of DBZ and gefitinib ( $n=8)$, as determined by staining tissue sections with H\&E. (B) The numbers of lung adenocarcinomas in the same mice as in $\mathbf{A}$. (C) H\&E and immunohistochemical staining of tumors from the same mice as in $\mathbf{A}$. The dot plots show the percentage of positive cells in the corresponding immunohistochemically stained sections. They correspond to the analysis of 5 fields (original magnification, $x 10$ ) per tumor. Scale bars: $100 \mu \mathrm{m}$; Inset scale bars: 25 $\mu \mathrm{m}$. Values correspond to the average \pm SEM. Statistical significance in A-C was determined by 1-way ANOVA followed by Tukey's post hoc test. ${ }^{*} P \leq 0.05$, ${ }^{* *} P \leq 0.01,{ }^{* *} P \leq 0.001,{ }^{* * *} P \leq 0.0001$. In panel $\mathbf{A}$, the comparison between gefitinib and DBZ single treatments was also significant. In panel $\mathbf{C}$, the difference between gefitinib alone and DBZ alone for HES1 and p-ERK staining was also significant $(P \leq 0.05$ and $P \leq 0.01$, respectively).

Taken together, our data indicate that the DBZ and gefitinib combination synergistically reduces the expression of HES1, a major driver in lung adenocarcinoma.

p-STAT3 directly binds to the HES1 promoter and inhibits its expression. Previous studies have shown a benefit of combining EGFR TKIs and Notch inhibitors in TKI-sensitive cells, but the underlying mechanism was not fully elucidated (15-17). On the basis of the EGFR-dependent HES1 decrease in EGFR ${ }^{\text {T790M/L858R_- }}$ expressing $\mathrm{CHO}$ cells upon incubation with the DBZ and gefitinib combination, we hypothesized that a common mechanism could be involved in the response to TKI treatment in TKI-sensitive and -resistant lung adenocarcinoma cells. An increase in the phos- 
A

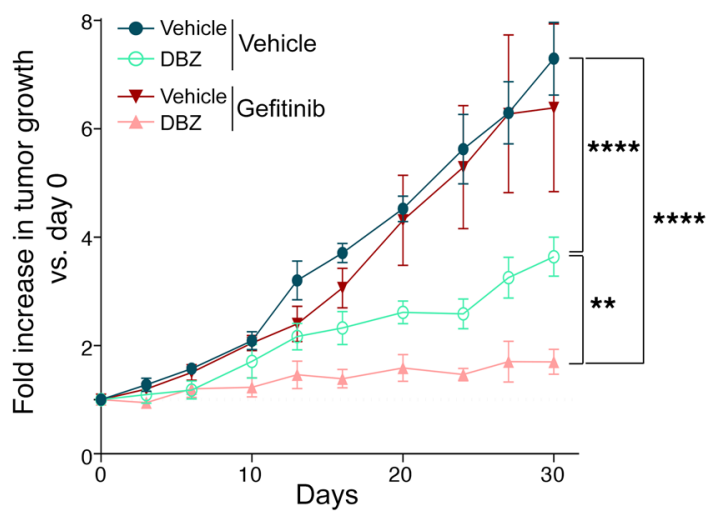

B
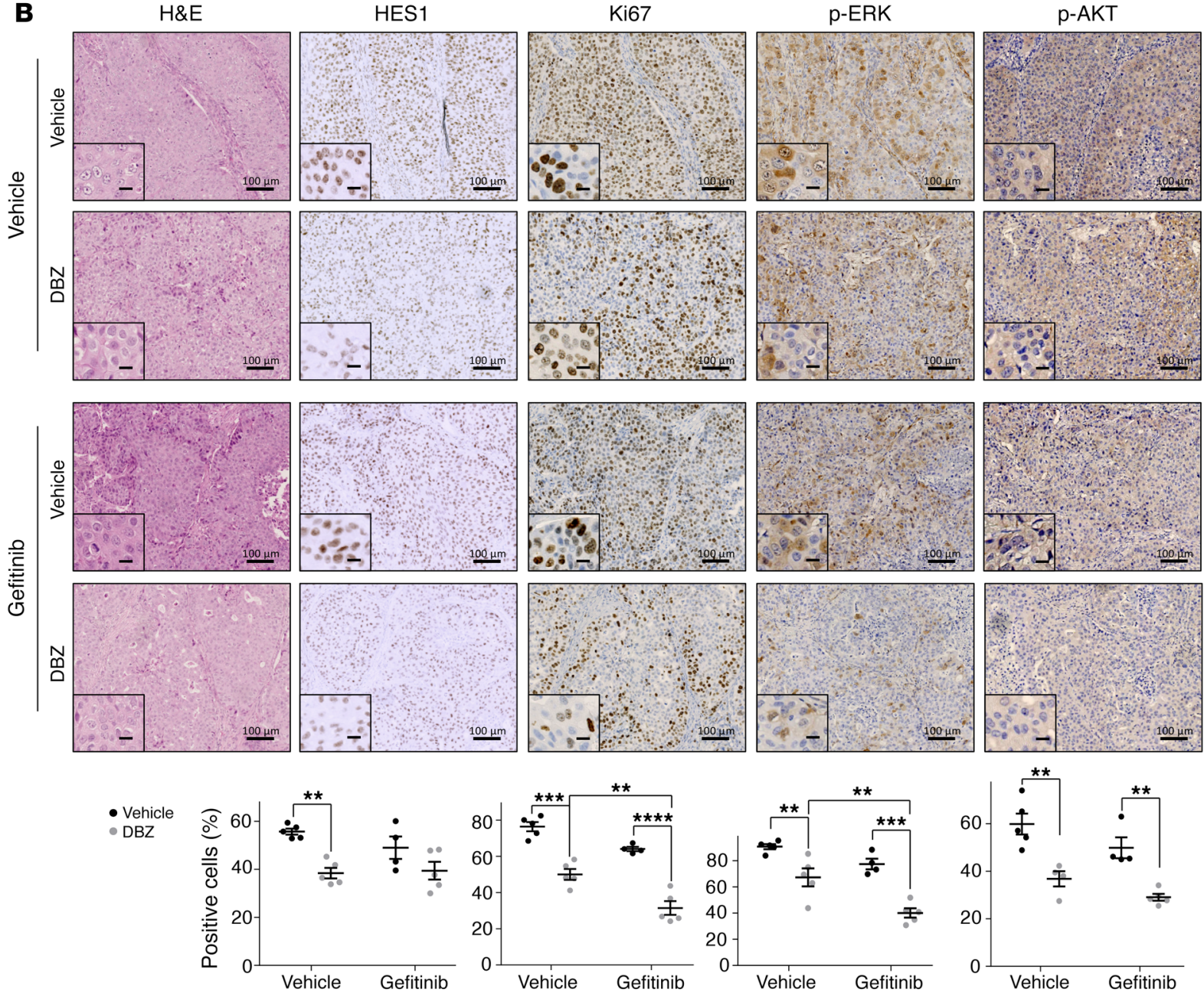

Figure 3. Notch inhibition sensitizes human ECFR ${ }^{T 790 M / L 858 R}$-driven lung adenocarcinoma to gefitinib. (A) Growth of PDX lung adenocarcinoma $E G F R^{T 790 M / L 858 R}$ implanted in the right flanks of nude mice treated with vehicle (methocel, $\left.n=5\right)$, DBZ $(n=5)$, gefitinib $(n=4)$, or a combination of DBZ and gefitinib $(n=5)$. The $y$ axis shows the fold increase in tumor size versus day 0 . (B) H\&E and immunohistochemical staining of tumors from the same mice as in $\mathbf{A}$. The dot plots show the percentage of positive cells in the corresponding immunohistochemically stained sections. For each treatment to the analysis of 5 fields (original magnification, $\times 10$ ) per mouse. Scale bars: $100 \mu \mathrm{m}$; Inset scale bars: $25 \mu \mathrm{m}$. Values correspond to the average \pm SEM. Statistical significance was determined by 2-way ANOVA in $\mathbf{A}$ and 1-way ANOVA in B followed by Tukey's post hoc test in both cases. ${ }^{* *} P \leq 0.01,{ }^{* * *} P \leq$ $0.001,{ }^{* * *} P \leq 0.0001$. In panel $\mathbf{A}$, the differences between gefitinib single treatment and DBZ or the combination were also significant $(P \leq 0.001$ and $P \leq 0.0001$, respectively). In panel B, the difference between vehicle and DBZ was also significant for all staining $(P \leq 0.01$ for HES1 and $P \leq 0.0001$ for Ki67, p-ERK, and p-AKT). Finally, the comparison between gefitinib and DBZ in Ki67 staining was also significant $(P \leq 0.05)$. 
A
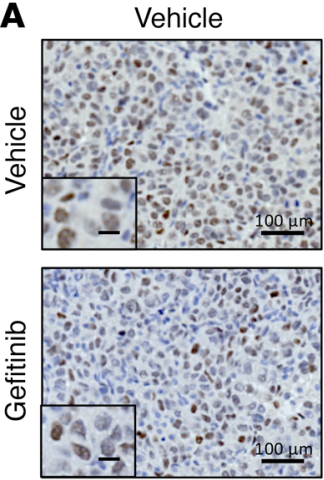

B
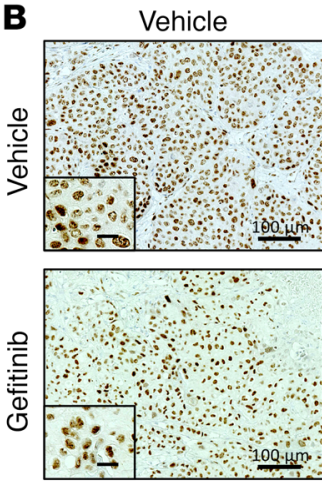

DBZ
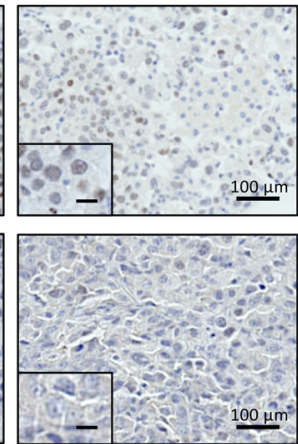

DBZ
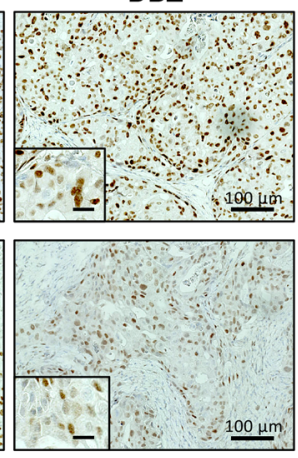
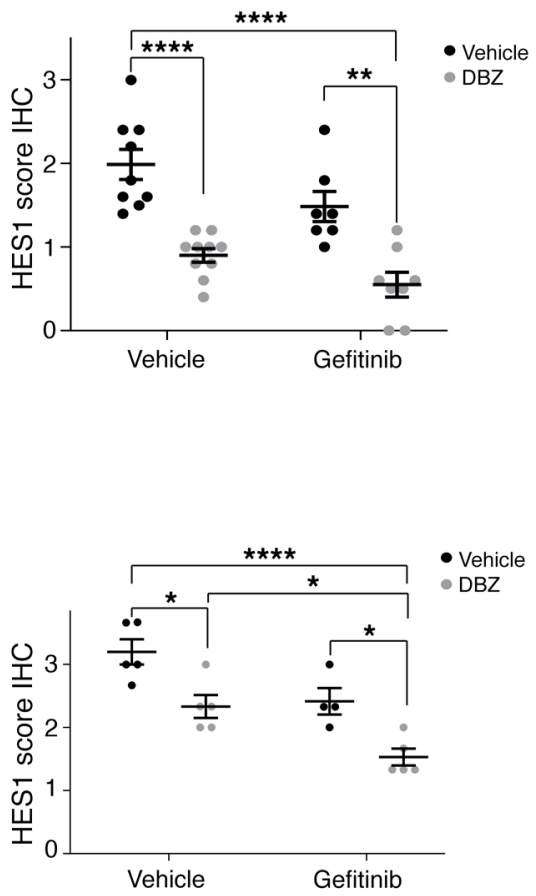

C

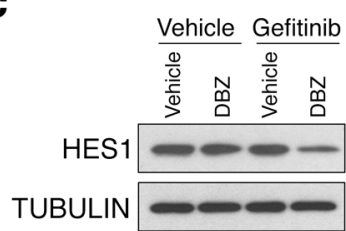

D

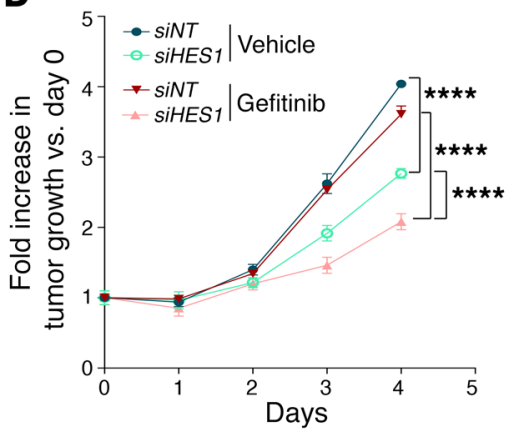

E

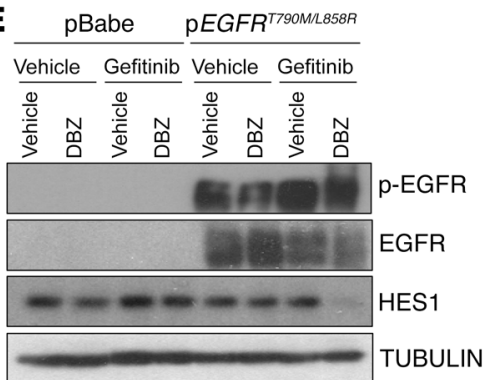

Figure 4. Combining EGFR TKIs and Notch inhibitors synergistically decreases HES1 expression. (A) Immunohistochemical staining of HES1 in tumors from EGFR ${ }^{T 790 M / L 858 R}$ mice treated with methocel (vehicle; $\left.n=9\right)$, DBZ $(n=10)$, gefitinib $(n=7)$, or with a combination of DBZ and gefitinib $(n=8)$. (B) Immunohistochemical staining of HES1 in tumors from EGFR ${ }^{T 790 M / L 858 R}$ PDX implanted in nude mice and treated with vehicle (methocel, $\left.n=5\right)$, DBZ $(n=5)$, gefitinib $(n=4)$, or the combination $(n=5)$. In $\mathbf{A}$ and $\mathbf{B}$, the dot plots show quantification of the intensity of the staining from the analysis of 5 fields (original magnification, $\times 10)$ per mouse. Scale bars: $100 \mu \mathrm{m}$; Inset scale bars: $25 \mu \mathrm{m}$. (C) Immunoblotting of the indicated proteins in PC9GR cells treated with vehicle (DMSO), DBZ (250 nM), and/or gefitinib $(1 \mu \mathrm{M})$. This is a representative image of 3 different experiments. (D) Proliferation of PC9CR cells transfected with a nontargeting siRNA (siNT) or siRNA targeting HES1 (siHES1) and treated with vehicle (DMSO) or gefitinib (15 nM) for 72 hours. The data are shown as mean \pm SEM ( $n=3$ in all groups). (E) Immunoblotting of the indicated proteins in CHO cells transfected with pBabe empty vector or $p E C F R^{T 790 M / L 858 R}$ and treated with vehicle (DMSO) or DBZ $(250 \mathrm{nM})$ and/or gefitinib $(1 \mu \mathrm{M})$. This is a representative image of 2 different experiments. Values correspond to the average \pm SEM. Statistical significance was analyzed by 1-way ANOVA in $\mathbf{A}$ and $\mathbf{B}$ and 2-way ANOVA in $\mathbf{D}$ followed by Tukey's post hoc test in all cases. ${ }^{*} P \leq 0.05,{ }^{* *} P \leq 0.01,{ }^{* * *} P \leq 0.0001$. In panel $\mathbf{A}$, the difference between gefitinib alone and DBZ alone is also significant $(P \leq 0.05)$. In panel $\mathbf{B}$, the difference between vehicle and gefitinib is also significant $(P \leq 0.05)$. In panel $\mathbf{D}$, the differences between vehicle and gefitinib alone or vehicle and siHES1/ gefitinib or gefitinib and siHES1 were also significant $(P \leq 0.01, P \leq 0.0001$, and $P \leq 0.0001$, respectively).

phorylation of STAT3 protein, dependent on both JAK and FGFR activities, is reported in sensitive lung adenocarcinoma cells upon treatment with first-generation (erlotinib) and second-generation (afatinib) TKIs (33-35); hence, we investigated whether this also occurred in TKI-resistant cells.

Indeed, analysis of STAT3 phosphorylation status in PC9GR cells showed an increase in p-STAT3 levels upon gefitinib treatment (Figure 5A). This effect was partially inhibited by cotreatment with PD173074 or ruxolitinib, pan-inhibitors of FGFR and JAK pathways, respectively. Moreover, the combination of both inhibitors reduced p-STAT3 to levels lower than in control nontreated cells (Supplemental Figure 4). Furthermore, we found that in the human HES1 and mouse Hes1 gene promoters, consensus binding sites for p-STAT3 (i.e., TTNNNNNAA) (36) are close to RBPJ sites (i.e., where the Notch transcription complex binds) (Supplemental Figure 5, A and B). To test whether p-STAT3 binds directly to the human HES1 promoter in PC9GR cells, we performed chromatin immunoprecipitation (ChIP) experiments using antibodies against p-STAT3 and against NOTCH1, which is known to bind to the HES1 promoter (positive control). NOTCH1 bound to the HES1 promoter, and this interaction was reduced by incubation with DBZ (Figure 5B). Importantly, p-STAT3 bound to the HES1 promoter only when cells were coincubated with gefitinib and DBZ (Figure 5B). To determine whether p-STAT3 binding was critical for HES1 downregulation (Figure 4C), we incubated PC9GR cells with the various drug combinations after siSTAT3 treatment that efficiently reduced both p-STAT3 and STAT3 expression (Figure 5C). Coincubation with gefitinib and DBZ strongly reduced HES1 protein levels in control siNT-treated cells (Figure 5C), but strikingly, the same cotreatment resulted in no appreciable change in HES1 levels in siSTAT3-treated cells (Figure 5C). 
A
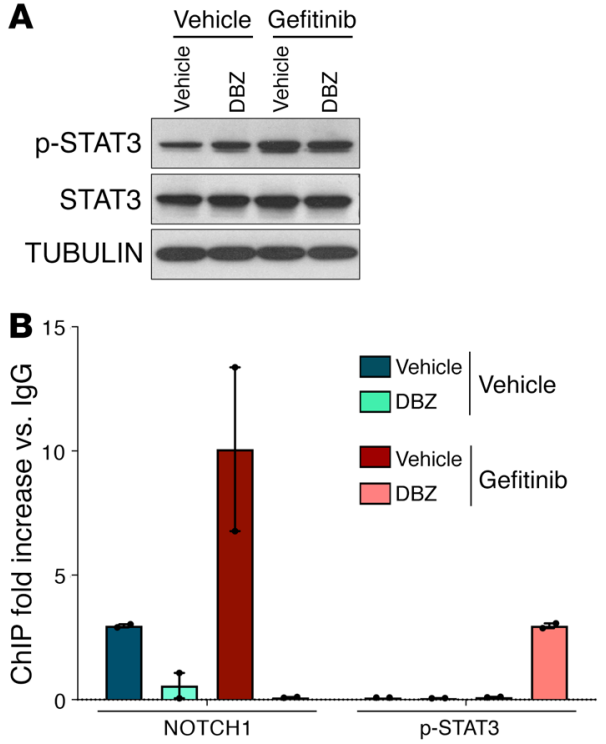

C

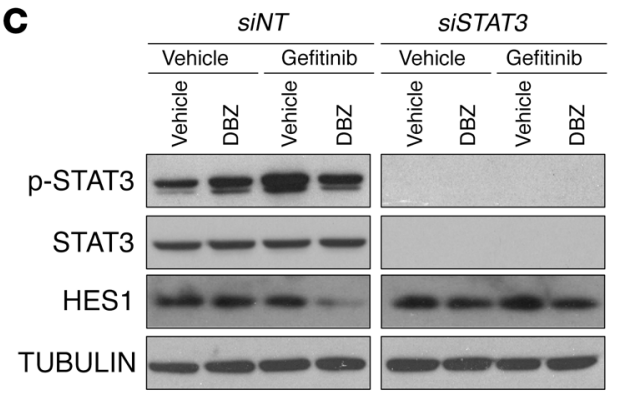

Altogether, these findings support the notion that p-STAT3 decreases HES1 protein levels by acting as a transcriptional repressor at the HES1 promoter.

Notch inhibition overcomes resistance to osimertinib in human lung adenocarcinoma cells harboring the EGFR ${ }^{C 7975}$ mutation. Because various TKIs increase p-STAT3 levels in lung adenocarcinoma cells (33-35), we asked whether the p-STAT3-dependent mechanism observed for gefitinib also applied to osimertinib. To this end, we used the PC9GROR cell line (previously generated from PC9GR cells) that is resistant to osimertinib and harbors the gatekeeper mutation $E G F R^{C 797 s}$ (18).

First, Western blot analysis of PC9GROR cells incubated with DBZ and/or osimertinib showed that p-STAT3 levels increased upon osimertinib treatment. Accordingly, the combination of DBZ and osimertinib reduced HES1 protein levels (Figure 6A).

To test whether DBZ resensitized $E G F R^{C 7975}$ mutant human lung adenocarcinoma cells to osimertinib in vivo, we grafted PC9GROR cells subcutaneously in mice, and 2 weeks later, we treated them with DBZ and/or osimertinib for 3 weeks. Body weight remained comparable in the different treatment groups (Supplemental Figure 6A). Osimertinib alone had no significant effect on growth of PC9GROR cell xenografts (Figure 6B), while it strongly inhibited the growth of PC9GR xenografts (Supplemental Figure 6B). Similarly, DBZ showed no effect on growth of PC9GROR cell xenografts, but importantly, tumor growth was strongly inhibited in mice treated with the osimertinib and DBZ combination (Figure 6B).
Figure 5. p-STAT3 directly binds to the HES1 promoter and inhibits its expression. (A) Immunoblotting of the indicated proteins in PC9GR cells treated with vehicle (DMSO), DBZ $(250 \mathrm{nM})$ and/or gefitinib $(1 \mu \mathrm{M})$. This is a representative image of 3 different experiments. (B) ChIP analysis of the binding of NOTCH1 and p-STAT3 to the HES1 promoter in PC9CR cells treated as in $\mathbf{A}$ ( $n=2$ per treatment). (C) Immunoblotting of the indicated proteins in PC9GR cells transfected with a nontargeting siRNA (siNT) or siRNA targeting STAT3 (sISTAT3) and treated with vehicle (DMSO) or DBZ $(250 \mathrm{nM})$ and/or gefitinib $(1 \mu \mathrm{M})$. This is a representative image of 2 different experiments.

This finding demonstrates that treatment with DBZ restores sensitivity to osimertinib in human lung adenocarcinoma cells harboring the $E G F R^{C 7975}$ mutation, confirming and extending our previous observations that DBZ sensitizes TKI-resistant tumors to TKIs.

Nirogacestat overcomes resistance to gefitinib in human lung adenocarcinoma cells harboring the EGFR ${ }^{T 790}$ mutation. To strengthen the translational impact of our work, we wanted to confirm the Notchinhibitor-sensitizing effect using a GSI under clinical trials. We chose nirogacestat because a recently finished phase II trial showed that it has promising effects in patients with desmoid tumors, is well tolerated, and can be used for long-term treatments (37).

We randomized mice with subcutaneous PC9GR cell xenografts in 6 treatment groups: (i) vehicle, (ii) DBZ, (iii) nirogacestat, (iv) gefitinib, (v) DBZ plus gefitinib, and (vi) nirogacestat plus gefitinib. Because gefitinib has some effect in PC9GR cells in vitro (Figure 4D), we used $10 \mathrm{mg} / \mathrm{kg}$ instead of the previously used dose of $20 \mathrm{mg} / \mathrm{kg}$. This lower concentration had a mild, nonsignificant effect on tumor growth compared with vehicle. Like in PC9GROR cells, the GSIs alone (DBZ and nirogacestat) did not have any effect. Conversely, gefitinib in combination with DBZ or nirogacestat strongly inhibited tumor growth (Figure 7A), as observed in mice harboring PDX and $E G F R^{T 790 M / L 858 R}$-driven tumors treated with the gefitinib and DBZ combination.

Moreover, Kaplan-Meier survival analysis of mice treated or not with nirogacestat and/or gefitinib showed that the survival rate was comparable in mice treated with vehicle, nirogacestat, or gefitinib alone, although it tended to be higher in the gefitinib group (Figure 7B). By contrast, the nirogacestat plus gefitinib combination increased survival compared with all other groups (median survival after treatment started: 24, 26.5, 32, and 39 days for vehicle, nirogacestat, gefitinib, and nirogacestat + gefitinib, respectively). For this analysis, we used only nirogacestat because at the used dose we could administer DBZ only for 5 weeks (26), while nirogacestat is well tolerated in patients for more than 2 years (37). As before, body weight was not significantly different in all groups during the experiment (Supplemental Figure 7).

These results show that the combination of gefitinib and nirogacestat increases the survival of mice xenografted with human lung adenocarcinoma cells that carry the $E G F R^{\mathrm{TT90M}}$ mutation conferring resistance to EGFR TKIs.

High HES1 protein levels correlate with poor progression-free survival and relapse in patients with EGFR-mutated lung adenocarcinoma treated with TKIs. Our findings showed that HES1 has a key role in the resistance of EGFR-driven lung adenocarcinoma to TKI therapy. To strengthen this observation, we analyzed the correlation between progression-free survival (PFS) and nuclear HES1 protein levels in 75 patients with lung adenocarcinoma harboring 
A
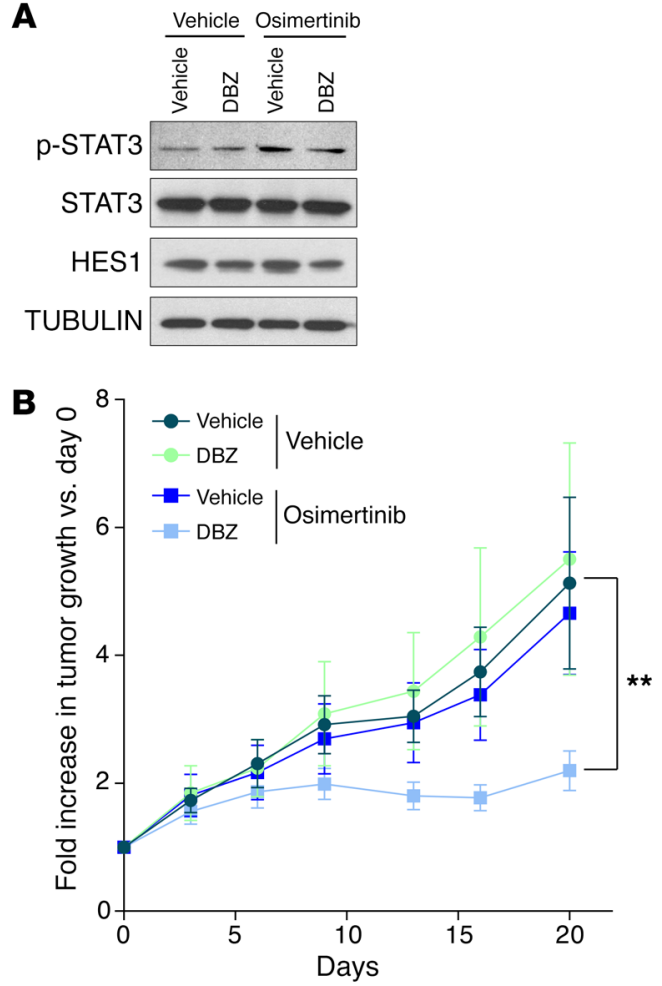

EGFR mutations and treated with TKIs. We found that patients with low nuclear HES1 expression had a median PFS of 14 months, whereas patients with high nuclear HES1 expression had a median PFS of 7 months (hazard ratio $2.77,95 \%$ CI 1.4-5.5, $P=0.006$ ) (Figure 7C). Moreover, analysis of HES1 protein in tumor biopsy samples from patients with lung adenocarcinoma harboring $E G F R$ activating mutations and treated with TKIs taken at diagnosis and after disease progression showed that HES1 nuclear levels were increased in samples obtained at relapse in 6 of the 7 patients $(P=$ 0.034) (Figure 7D and Supplemental Figure 8).

These findings extend our previous study (14) and suggest a crucial role for HES1 in the relapse of patients with EGFR-driven lung adenocarcinoma under treatment with TKIs.

\section{Discussion}

In this study, we have extended the role of HES1 as a crucial mediator of the oncogenic activity of the Notch pathway in lung adenocarcinoma and uncover its crucial role in resistance to EGFR TKIs.

We first observed that in EGFR-driven lung adenocarcinoma, treatment with GSIs produces a decrease in HES1 expression concomitant with a decrease in $\mathrm{p}$-ERK protein levels. This is consistent with the HES1-induced repression of DUSP1 that, in turn, would increase p-ERK levels, as previously described in KRAS-driven lung adenocarcinoma $(13,14)$. Hence, we assume this is also the main mechanism for the antitumor effect of Notch inhibition as single treatment in EGFR-driven tumors.

Next, as a proof of concept of resensitizing cells with EGFR gatekeeper mutations to TKIs upon Notch inhibition, we found that murine and human EGFR-driven lung tumors harboring the $E G F R^{T 790 M}$ gatekeeper mutation are resensitized to gefitinib upon combination with the GSI DBZ. Concomitantly, we found that
Figure 6. Notch inhibition sensitizes EGFR ${ }^{\text {C7975 }}$ cells to osimertinib. (A) Immunoblotting of the indicated proteins in PC9GROR cells treated with vehicle (DMSO), DBZ (250 nM) and/or osimertinib (250 nM). (B) PC9GROR cells were injected subcutaneously in the right flank of nude mice. The mice were then treated with vehicle (methocel, $n=8)$, DBZ $(n=8)$, osimertinib $(n=8)$, or the combination of DBZ and osimertinib $(n=7)$. The $y$ axis shows the tumor growth fold increase versus day 0 . Values correspond to the average \pm SEM. Statistical significance was determined by 2-way ANOVA followed by Tukey's post hoc test. ${ }^{* *} P \leq 0.01$. In panel $\mathbf{B}$, the differences between DBZ and the combination or between osimertinib and the combination were also significant $(P \leq 0.01$ and $P \leq 0.05$, respectively).

p-AKT and p-ERK were also further decreased upon combined treatment with gefitinib and DBZ, compared with DBZ single treatment. It is reported that HES1 represses PTEN with increasing AKT activity in T-ALL (31) and HES1 also increases p-ERK levels in lung adenocarcinoma (30). Interestingly, we found in both transgenic and PDX-preclinical mouse models a decreased expression of HES1 levels in the combination of DBZ and gefitinib compared with DBZ single treatment. Using loss-of-function analysis, we also found that cells are sensitized to gefitinib in the absence of HES1. Why does HES1 loss of function promote this sensitivity beyond p-AKT and p-ERK? For instance, it is reported in T-ALL that HES1 directly represses the BBC3 gene (i.e., PUMA), an inducer of apoptosis (38), so it is tempting to speculate that HES1 could also repress the $B B C 3$ gene or other important apoptotic inducers in this context, for example BCL2L11 (i.e., BIM), which is crucial in gefitinib-induced cell death (39-41).

The $E G F R^{T 790 M}$ mutation does not totally inhibit the binding of gefitinib to the EGFR protein $(8,9)$, and moreover, EGFR was needed to further lower HES1 expression in the cotreatment with gefitinib and DBZ compared with DBZ alone. Because an increase in active STAT3 upon treatment with both first- and secondgeneration TKIs has been reported (33-35), we hypothesized that this feature could explain the decreased levels of HES1 in our experimental setting. Indeed, gefitinib treatment of our $E G F R^{T 790 M}$ mutant cell model increased p-STAT3 protein levels in an FGFRand JAK-activity-dependent manner. Also, a direct recruitment of p-STAT3 onto the HES1 promoter was detected by ChIP only when gefitinib was combined with GSI. And finally, loss of function of STAT3 maintains HES1 protein in the cotreated cells at similar levels to those in cells treated with DBZ alone. Our data show that p-STAT3 requires concomitant inhibition of NOTCH processing to repress HES1 expression effectively, probably because the NOTCH transcriptional complex binds more efficiently than p-STAT3. A previous report showed that erlotinib treatment increased the Notch pathway after several days in EGFR-driven lung adenocarcinoma TKI-sensitive cells (16). We do not see such induction and this discrepancy could be due to the different treatment kinetics and/or the resistant background of PC9GR cells. Our data are in accordance with the work developed by others, both in the function of p-STAT3 as a transcriptional repressor (42) and in its tumor suppressive role in some types of cancer, including prostate (43), glioblastoma (44), and importantly, KRASdriven lung adenocarcinoma (45). In light of these data, STAT3 inhibitors currently in clinical trials (46) should be used with caution, at least in those tumors where the Notch pathway, and hence HES1, play a protumorigenic role, as in lung adenocarcinoma. 
A
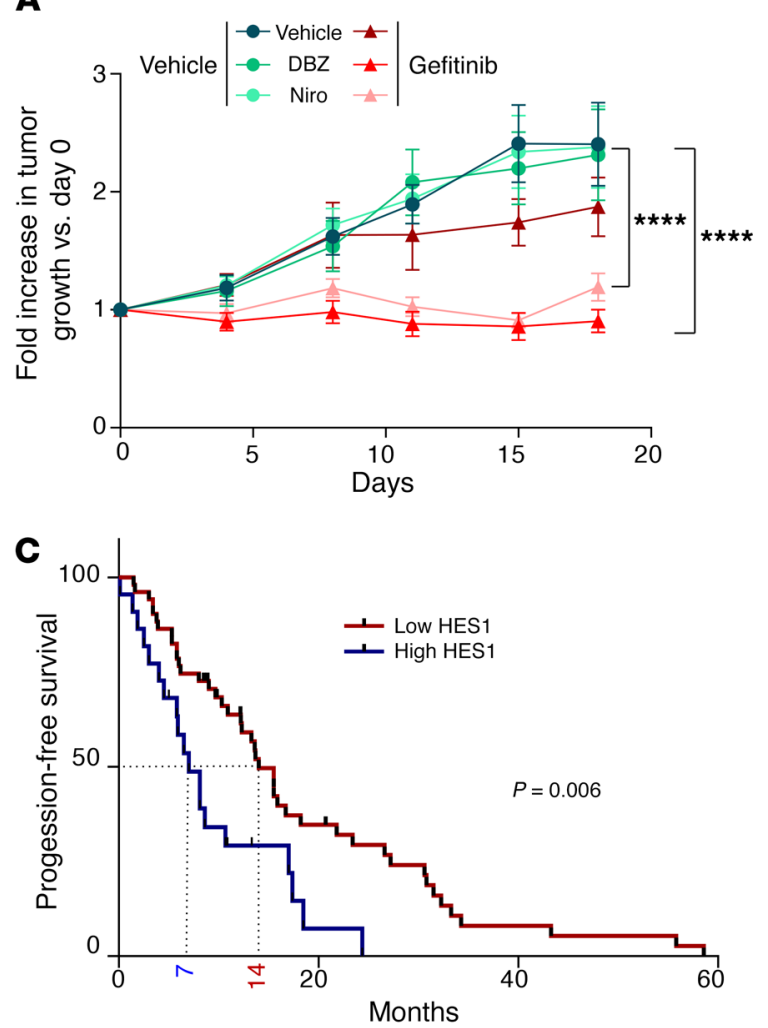

B

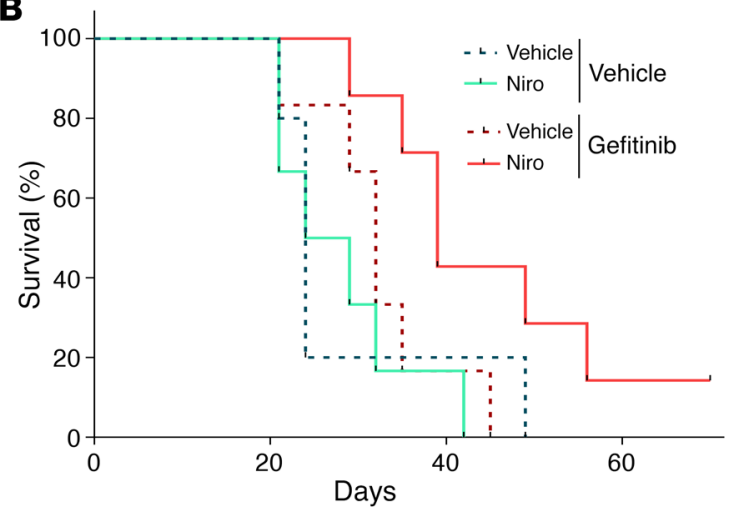

D

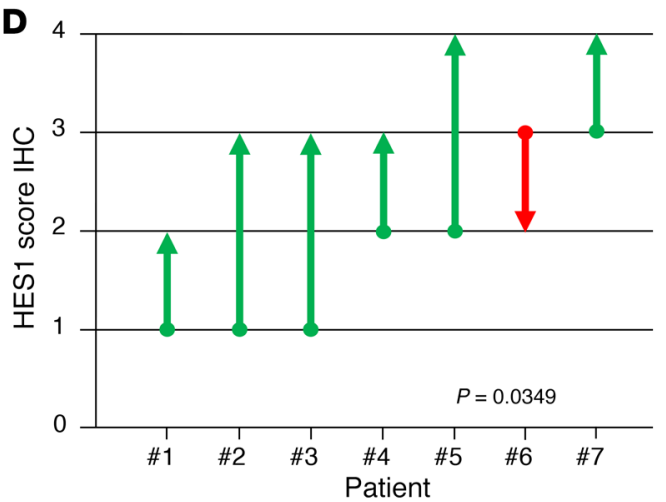

Figure 7. High HES1 protein levels correlate with poor progression-free survival and relapse in EGFR-mutated lung adenocarcinoma patients under TKI treatment. (A) PC9GR cells were injected subcutaneously into nude mice. The mice were then treated with vehicle (methocel, $n=6)$, DBZ $(n=6)$, nirogacestat $(n=6)$, gefitinib $(n=6)$, or the combination of DBZ and gefitinib $(n=7)$, or the combination of nirogacestat and gefitinib ( $n=7)$. The $y$ axis shows the tumor growth fold increase versus day 0 and the $x$ axis the days after treatment. Values correspond to the average \pm SEM. Statistical significance was determined by 2-way ANOVA followed by Tukey's post hoc test. ${ }^{* * *} P \leq 0.0001$. The differences between DBZ and the combination DBZ/gefitinib or nirogacestat/gefitinib were also significant $(P \leq 0.0001$ and $P \leq 0.001$, respectively), as was the difference between nirogacestat and the combination DBZ/gefitinib or nirogacestat/gefitinib ( $P \leq 0.0001$ for both). The difference between gefitinib alone and the combination DBZ plus gefitinib was also significant $(P \leq 0.01)$. (B) PC9GR cells were injected subcutaneously in nude mice. The mice were then treated with vehicle (methocel, $n=5)$, nirogacestat $(n=6)$, gefitinib $(n=6)$, or the combination of DBZ and gefitinib $(n=7)$. The $y$ axis shows the percentage of surviving animals and the $x$ axis the days after treatment. Statistical significance was determined by the Gehan-Breslow-Wilcoxon test. Vehicle vs. gefitinib $(P=0.3)$, vehicle vs. nirogacestat $(P=$ $0.93)$, vehicle vs. the combination $(P=0.02)$, gefitinib vs. the combination $(P=0.05)$, and nirogacestat vs. the combination $(P=0.02)$. (C) Progression-free survival of EGFR TKI-treated patients with EGFR-mutated lung adenocarcinoma $(n=75)$ according to HES1 expression assessed by immunohistochemical staining (low HES1 = 0-2.50 HES1 score; high HES1 = 2.51-5.00 HES1 score). Statistical significance was determined by the Gehan-Breslow-Wilcoxon test. (D) Representation of the change in HES1 immunohistochemical staining intensity score in patient samples before treatment (dots) and after relapse (arrowheads). Statistical significance was determined by paired 2-tailed Student's $t$ test.

Our findings provide a proof of concept for sensitizing lung adenocarcinoma cells with gatekeeper mutations to TKIs by inhibiting $\gamma$-secretase. To extend and validate our findings, we performed an additional assay in lung adenocarcinoma cells with the osimertinib-resistant gatekeeper mutation $E G F R^{C 797 S}$. The relevance of this experiment relies in results from a recent phase III clinical trial showing that the PFS of patients with EGFR mutations when treated with osimertinib as a first-line treatment was significantly longer than those patients treated with first-line gefitinib or erlotinib (47). Hence, most probably the use of osimertinib as first-line treatment in EGFR-mutated lung adenocarcinoma patients will begin soon. Our data show that osimertinib treatment in lung adenocarcinoma cells harboring the EGFR ${ }^{C 7975}$ mutation also induced p-STAT3 and inhibited HES1 expression, when combined with DBZ. More importantly, it also strongly inhibited tumor growth in the same cells in vivo. Hence, we predict a scenar- io in which osimertinib will bind poorly to EGFR due to the lack of covalent binding induced by the EGFR ${ }^{C 7975}$ gatekeeper mutation, but the binding still would be sufficient to promote changes similar to those we found in $E G F R^{T 790 M}$ cells treated with gefitinib and these changes will also be exacerbated in the presence of Notch inhibition. Our results invoke further investigation regarding the effect of the Notch pathway in the drug-tolerant state (48) in lung adenocarcinoma cells under osimertinib treatment and this is currently an important area of study in the laboratory.

Overall, the mechanistic data described above depict a role for HES1 in relapse after EGFR TKI therapy, and accordingly, we show a negative correlation between HES1 expression and PFS as well as an increase in HES1 expression upon disease progression in EGFR-mutated patients being treated with TKIs. Our results are in accordance with a recent publication that shows a negative correlation between HES1 mRNA levels and PFS in 
a cohort of 64 EGFR-mutated non-small cell lung carcinoma patients treated with TKIs (49).

Our findings might be very relevant for EGFR-driven lung adenocarcinoma patients with gatekeeper mutations such as $E G F R^{C 7975}$ that relapses after osimertinib, where treatment possibilities are mainly limited to conventional therapies, since immune checkpoint inhibitors are mostly ineffective in this context. Our findings warrant the development of a phase I clinical trial to demonstrate the efficacy of the GSI-TKI drug combination in patients. Interestingly, a phase I/II trial in 16 patients that combined the TKI erlotinib and a GSI from Roche, RO4929097, showed that this combination was safe and feasible in lung adenocarcinoma patients (50). Because the side effects associated with erlotinib are higher than those with osimertinib (47), a combination of osimertinib and GSIs is also likely to be safe in patients. For instance, nirogacestat showed long-term efficacy and is well tolerated in patients (37), and moreover, we demonstrated here that it sensitizes human lung adenocarcinoma cells harboring gatekeeper mutations against TKIs.

\section{Methods}

Mice. Tet-on-EGFR ${ }^{T 790 M / L 858 R}$ and CCSP-rtTA mice were described previously $(22,23)$. For in vivo PC9GR and PC9GROR lung adenocarcinoma cell tumor growth assays, 6-week-old, female athymic NudeFoxn1 mice (Envigo) were injected subcutaneously in the flank with $3.5 \times 10^{6}$ PC9GR or PC9GROR cells. Drug treatments were started when tumors were $200 \mathrm{~mm}^{3}$. In Kaplan-Meier analyses, mice were killed when tumors reached $1200 \mathrm{~mm}^{3}$.

Western blotting. Western blotting was performed as previously described (14). The following antibodies were used for the analysis: N1ICD (4147, Cell Signaling Technology, 1:500 dilution); HES1 (11988, Cell Signaling Technology, 1:1000 dilution); NOTCH1 (3608, Cell Signaling Technology, 1:1000 dilution); NOTCH2 (5732, Cell Signaling Technology, 1:1000 dilution); NOTCH3 (5276, Cell Signaling Technology, 1:1000 dilution); p-STAT3 (9145, Cell Signaling Technology, 1:1000 dilution); total STAT3 (610189, BD, 1:1000 dilution); and tubulin (T9026, Sigma-Aldrich, 1:2000 dilution). Secondary antibodies were either horseradish peroxidase-conjugated anti-rabbit (7077, Cell Signaling Technology, 1:10,000 dilution) or anti-mouse (7076, Cell Signaling Technology, 1:10,000 dilution). Antibody binding was detected by chemiluminescence using the ECL detection system (GE Healthcare) or ECL Plus (for N1ICD) (GE Healthcare).

Treatments in mice. Dibenzazepine (DBZ) (Syncom) and nirogacestat (MedChemExpress) were administered 4 days per week (3.3 and $50 \mathrm{mg} / \mathrm{kg} /$ day, respectively) by intraperitoneal (i.p.) injection or gavage, respectively. Gefitinib and osimertinib (CliniSciences) were administered by gavage 4 days per week $(20 \mathrm{mg} / \mathrm{kg} /$ day $)$ and 5 days per week (5 mg/kg/day), respectively. Antibodies against NOTCH1 (NRR1) and NOTCH3 (NRR3) were administered by i.p. injection: $\mathrm{NRR} 1$ at $5 \mathrm{mg} / \mathrm{kg}$ /day every 5 days and NRR3 at $15 \mathrm{mg} / \mathrm{kg}$ /day every Monday and Thursday (Genentech).

Histopathology and IHC. Lung lobes were fixed, embedded in paraffin, and stained with hematoxylin and eosin (H\&E) or used for IHC. Tumor area and total lung area were measured using ImageJ software (NIH). For pathological analysis of H\&E-stained sections, classical cytological and architectural features (such as invasion or high mitotic rate) were examined in-house by a pathologist. For IHC, the following antibodies were used: rabbit monoclonal anti-HES1 (1:1000 dilution, 11988, Cell Signaling Technology); rat monoclonal anti-Ki67 (1:100 dilution, TEC-3, Agilent); rabbit polyclonal anti-phospho-p44/42 Erk1/Erk2 (Thr202/Tyr204 and Thr185/Tyr187, respectively) (1:25 dilution, 9101, Cell Signaling Technology); and rabbit monoclonal anti-phospho-Akt1 (Ser473) (1:175 dilution, clone EP2109Y, Novus Biologicals). For each tumor, 5 fields ( $\times 10$ magnification) were scored using Image J software. Murine and clinical samples were scored for intensity ( 0 for the lowest intensity and 5 for the highest).

Cell culture and transfection reagents. PC9GR (resistant to gefitinib), and PC9GROR (resistant to gefitinib and osimertinib) were obtained from the Yarden lab (18). The siRNA control (nontargeting, siNT) and that against HES1 (siHES1) or STAT3 (siSTAT3) (Dharmacon) were transfected at $20 \mathrm{nM}$ with Dharmafect1 following the manufacturer's instructions.

For Western blotting, RNA-Seq, or ChIP, cells were treated with DBZ (250 nM) (or DMSO as vehicle), gefitinib $(1 \mu \mathrm{M})$, osimertinib (250 nM), PD173074 $(2 \mu \mathrm{M})$, or ruxolitinib $(0.25 \mu \mathrm{M})$; the last $2 \mathrm{~mol}-$ ecules were obtained from CliniSciences. For the siRNA proliferation assay, cells were treated with gefitinib (15 nM) (or DMSO as vehicle). The cells were fixed at various time points and stained with sulforhodamine B. Absorbance was measured at $560 \mathrm{~nm}$ in a microplate reader (Glomax, Promega).

RNA-Seq. RNA was sequenced by Fasteris using next-generation DNA sequencing based on Illumina technology. The RNA-Seq data were deposited in the NCBI's Gene Expression Omnibus (GEO GSE117846).

Reads were aligned against the Ensembl Homo sapiens genome assembly (GRCh38). Read counts were extracted from the STAR output file with HTSeq and only the protein-coding genome features were taken into account in the final count matrix.

Sample count normalization was realized by summing read counts for each sample $\left(s_{i}, i=1, \ldots 12\right)$, computing a first factor for each sample $\left(f_{i}=s_{i} /\right.$ median $\left._{j=1, \ldots, 12}\left[s_{j}\right]\right)$. These factors were normalized such that the product of all the normalized factors $g_{i}$ is equal to 1 :

$$
g_{i}=\frac{f_{i}}{\sqrt[1 / 12]{\prod_{j=1, \ldots, 12} f_{j}}}
$$

(Equation 1)

Finally, each column (each sample) of the read count matrix was divided by the corresponding $g a_{i}$. We analyzed for gene set enrichment analysis (19) using The Molecular Signatures Database Hallmark Gene Set Collection (20).

ChIP. The chromatin was prepared as described previously (51). We used the ChIP-Adem-Kit and ChIP DNA Prep Adem-Kit (Ademtech) for ChIP and DNA purification, respectively, on an AutoMag robot, according the manufacturer's instructions. The anti-NOTCH1 antibody was purchased from Abcam (catalog ab27525) and the antiphospho-STAT3 from Cell Signaling Technology (catalog 9145). The immunoprecipitated DNA was analyzed by PCR using the primers PromHES1 Fw, GAAGGCAATTTTTCCTTTTTC and PromHES1 Rev, AAGTTCCCGCTCAGACTTTAC.

PDX model. The PDX model was generated in the Paz-Ares laboratory at the Instituto de Biomedicina de Sevilla (IBIS). The tumor had a TNM of T2a N1 MO. Tumor sections $\left(0.5 \mathrm{~mm}^{3}\right)$ were implanted into the right flanks of 6-week-old, female athymic Nude-Foxn1 
mice (Envigo) and after 2 weeks the mice were randomized and the treatments started.

Patients. Tumors were analyzed from patients with EGFR mutations and treated with EGFR TKIs. Seventy-five patients were being treated at Toulouse University Hospital (52), and 4 had participated in the MOSCATO (NCT01566019) or MATCH-R (NCT02517892) clinical trials at the Institut Gustave Roussy.

Statistics. Unless otherwise specified, the data are presented as means \pm SEM. One-way analysis of variance (ANOVA) followed by Tukey's post hoc test was performed to assess the significance of expression levels in IHC, as well as to determine the differences among groups for changes in size of tumors or animal weight. In Figures 3A, 4D, 6B, and 7A, a repeated-measures 2-way ANOVA followed by Tukey's post hoc test was performed. In Figure 7, B and C, we analyzed the results with the Gehan-Breslow-Wilcoxon test. Hazard ratios were calculated using the Mantel-Haenszel test. In Figure 7D, data were analyzed by paired 2-tailed Student's $t$ test. Samples (cells or mice) were allocated to their experimental groups according to their predetermined type (cell type or mouse treatment). Investigators were blinded to the experimental groups in the analysis of data presented in Figure 1, C and D; Figure 2, A-C; Figure 3B; Figure 4, A and B; Figure $5 \mathrm{D}$; and Figure 7, C and D. The investigators were not blinded in the remaining analyses. $P \leq 0.05$ was considered significant. ${ }^{*} P \leq 0.05,{ }^{* *} P$ $\leq 0.01,{ }^{* * *} P \leq 0.001,{ }^{* * *} P \leq 0.0001$.

Study approval. Animal procedures were performed according to protocols approved by the French National Committee of animal care.

All patients had signed an informed consent form permitting analyses of tissues. This study was approved by the Committee for the Protection of Persons of each institution and by the French National Agency for Medicines and Health Products Safety (ANSM).

\section{Author contributions}

EBM designed and performed most experiments, analyzed data, and wrote the manuscript. SB performed the experiments for RNA-Seq and, together with LP, performed some experiments. $\mathrm{MM}, \mathrm{MG}$, and AG performed some in vivo treatments. EF performed ChIP experiments. IF and LPA developed and performed the PDX experiments. XQ, JLP, OC, JCS, JM, and GF performed experiments on clinical samples. MC performed the immunohistochemical analysis of mouse tumors. HPM and EC supervised the experiments with STAT3. YG and NP performed some immunohistochemistry experiments. KKW supervised the experiments on $E G F R^{T 790 M / L 858 R}$ mice. YY supervised the work with PC9 resistant cell lines. AT and JC performed the RNA-Seq analysis. CWS supervised the experiments with NRR1 and NRR3 antibodies. AM designed and supervised the study, secured funding, analyzed data, and wrote the manuscript. All authors discussed the results and commented on the manuscript.

\section{Acknowledgments}

We thank Daniel Herranz, Laurent Le Cam, Daniel Fisher, Hélène Tourriere, and Manuel Serrano for helpful discussion and critical reading of the manuscript. Elisabetta Andermarcher professionally edited the manuscript. We thank Dom Helmlinger for technical help with the RNA-Seq. We thank Simon Cabello-Aguilar for his help uploading the RNA-Seq into the NCBI's GEO database. We thank the IRCM's animal facility unit members for their outstanding work. We thank the immunohistochemistry technical service of Cell Signaling Technology for their help and the immunohistochemistry platform at CNIO for their work. EBM was supported by a contract from Fondation de France. SB was supported by a fellowship from the French Ministry of Education and Research. MM is supported by a fellowship from Fondation ARC. Work in AM's lab is supported by the Fondation ARC (PJA 20131200405), the European Commission (CIG631390), the Institut de Cancer de Montpellier (ICM) Fondation, and the Institut National du Cancer (INCa 9257 and SIRIC Montpellier Cancer Grant INCa Inserm DGOS 12553). The funders had no role in study design, data collection and analysis, decision to publish, or preparation of the manuscript.

Address correspondence to: Antonio Maraver, Oncogenic pathways in lung cancer, Institut de Recherche en Cancérologie de Montpellier, Inserm U1194 - Université Montpellier -ICM, Campus Val d'Aurelle, 208 Rue des Apothicaires, F-34298 Montpellier Cedex 5, France. Phone: 33.0.467.612.395; Email: antonio. maraver@inserm.fr.
1. Herbst RS, Heymach JV, Lippman SM. Lung cancer. N Engl J Med. 2008;359(13):1367-1380.

2. Rosell R, Bivona TG, Karachaliou N. Genetics and biomarkers in personalisation of lung cancer treatment. Lancet. 2013;382(9893):720-731.

3. Reck M, Heigener DF, Mok T, Soria JC, Rabe KF. Management of non-small-cell lung cancer: recent developments. Lancet. 2013;382(9893):709-719.

4. Tan CS, Gilligan D, Pacey S. Treatment approaches for EGFR-inhibitor-resistant patients with non-small-cell lung cancer. Lancet Oncol. 2015;16(9):e447-e459.

5. Jänne PA, et al. AZD9291 in EGFR inhibitorresistant non-small-cell lung cancer. $N$ Engl J Med. 2015;372(18):1689-1699.

6. Thress KS, et al. Acquired EGFR C797S mutation mediates resistance to AZD9291 in non-small cell lung cancer harboring EGFR T790M. Nat Med. 2015;21(6):560-562.

7. Le X, et al. Landscape of EGFR-dependent and -independent resistance mechanisms to osimertinib and continuation therapy beyond progression in EGFR-mutant NSCLC. Clin Cancer Res. 2018;24(24):6195-6203.

8. Yun $\mathrm{CH}$, et al. The T790M mutation in EGFR kinase causes drug resistance by increasing the affinity for ATP. Proc Natl Acad Sci U S A. 2008;105(6):2070-2075.

9. Yoshikawa S, et al. Structural basis for the altered drug sensitivities of non-small cell lung cancerassociated mutants of human epidermal growth factor receptor. Oncogene. 2013;32(1):27-38.

10. Bray SJ. Notch signalling in context. Nat Rev Mol Cell Biol. 2016;17(11):722-735.

11. Takebe N, et al. Targeting Notch, Hedgehog, and Wnt pathways in cancer stem cells: clinical update. Nat Rev Clin Oncol. 2015;12(8):445-464.

12. Licciulli S, et al. Notch1 is required for Krasinduced lung adenocarcinoma and controls tumor cell survival via p53. Cancer Res.
2013;73(19):5974-5984

13. Baumgart A, et al. Opposing role of Notch1 and Notch2 in a Kras(G12D)-driven murine non-small cell lung cancer model. Oncogene. 2015;34(5):578-588.

14. Maraver A, et al. Therapeutic effect of $\gamma$-secretase inhibition in KrasG12V-driven non-small cell lung carcinoma by derepression of DUSP1 and inhibition of ERK. Cancer Cell. 2012;22(2):222-234.

15. Konishi J, Yi F, Chen X, Vo H, Carbone DP, Dang TP. Notch3 cooperates with the EGFR pathway to modulate apoptosis through the induction of bim. Oncogene. 2010;29(4):589-596.

16. Arasada RR, Amann JM, Rahman MA, Huppert SS, Carbone DP. EGFR blockade enriches for lung cancer stem-like cells through Notch3-dependent signaling. Cancer Res. 2014;74(19):5572-5584.

17. Hu S, et al. Antagonism of EGFR and Notch limits resistance to EGFR inhibitors and radiation by decreasing tumor-initiating cell frequency. Sci 
Transl Med. 2017;9(380):eaag0339.

18. Mancini M, et al. An oligoclonal antibody durably overcomes resistance of lung cancer to thirdgeneration EGFR inhibitors. EMBO Mol Med. 2018;10(2):294-308.

19. Subramanian A, et al. Gene set enrichment analysis: a knowledge-based approach for interpreting genome-wide expression profiles. Proc Natl Acad Sci U S A. 2005;102(43):15545-15550.

20. Liberzon A, Birger C, Thorvaldsdóttir H, Ghandi M, Mesirov JP, Tamayo P. The Molecular Signatures Database (MSigDB) hallmark gene set collection. Cell Syst. 2015;1(6):417-425.

21. Arteaga CL, Engelman JA. ERBB receptors: from oncogene discovery to basic science to mechanism-based cancer therapeutics. Cancer Cell. 2014;25(3):282-303.

22. Li D, et al. Bronchial and peripheral murine lung carcinomas induced by T790M-L858R mutant EGFR respond to HKI-272 and rapamycin combination therapy. Cancer Cell. 2007;12(1):81-93.

23. Tichelaar JW, Lu W, Whitsett JA. Conditional expression of fibroblast growth factor-7 in the developing and mature lung.J Biol Chem. 2000;275(16):11858-11864.

24. Zheng Y, et al. A rare population of CD24(+) ITGB 4(+)Notch(hi) cells drives tumor propagation in NSCLC and requires Notch3 for selfrenewal. Cancer Cell. 2013;24(1):59-74.

25. Choy L, et al. Constitutive NOTCH3 signaling promotes the growth of basal breast cancers. Cancer Res. 2017;77(6):1439-1452.

26. Rivera-Torres J, et al. Targeting $\gamma$-secretases protect against angiotensin II-induced cardiac hypertrophy. JHypertens. 2015;33(4):843-850.

27. Wu Y, et al. Therapeutic antibody targeting of individual Notch receptors. Nature. 2010;464(7291):1052-1057.

28. van Es JH, et al. Notch/gamma-secretase inhibition turns proliferative cells in intestinal crypts and adenomas into goblet cells. Nature. 2005;435(7044):959-963.

29. Day CP, Merlino G, Van Dyke T. Preclinical mouse cancer models: a maze of opportunities and challenges. Cell. 2015;163(1):39-53.

30. Maraver A, Serrano M. Notching up a new therapeutic strategy for non-small cell lung carcinoma (NSCLC). Oncotarget. 2012;3(9):917-918.

31. Palomero T, et al. Mutational loss of PTEN induces resistance to NOTCH1 inhibition in T-cell leukemia. Nat Med.2007;13(10):1203-1210.

32. Lo HW, et al. Nuclear interaction of EGFR and STAT3 in the activation of the iNOS/NO pathway. Cancer Cell. 2005;7(6):575-589.

33. Kim SM, et al. Activation of IL-6R/JAK1/STAT3 signaling induces de novo resistance to irreversible EGFR inhibitors in non-small cell lung cancer with T790M resistance mutation. Mol Cancer Ther. 2012;11(10):2254-2264.

34. Lee HJ, Zhuang G, Cao Y, Du P, Kim HJ, Settleman J. Drug resistance via feedback activation of Stat 3 in oncogene-addicted cancer cells. Cancer Cell. 2014;26(2):207-221.

35. Codony-Servat C, et al. Activation of signal transducer and activator of transcription 3 (STAT3) signaling in EGFR mutant non-small-cell lung cancer (NSCLC). Oncotarget. 2017;8(29):47305-47316.

36. Darnell JE. STATs and gene regulation. Science. 1997;277(5332):1630-1635.

37. Kummar S, et al. Clinical activity of the $\gamma$-secretase inhibitor PF-03084014 in adults with desmoid tumors (aggressive fibromatosis). JClin Oncol. 2017;35(14):1561-1569.

38. Schnell SA, et al. Therapeutic targeting of HES1 transcriptional programs in T-ALL. Blood. 2015;125(18):2806-2814.

39. Gong Y, et al. Induction of BIM is essential for apoptosis triggered by EGFR kinase inhibitors in mutant EGFR-dependent lung adenocarcinomas. PLoS Med. 2007;4(10):e294.

40. Costa DB, et al. BIM mediates EGFR tyrosine kinase inhibitor-induced apoptosis in lung cancers with oncogenic EGFR mutations. PLoS Med. 2007;4(10):1669-1679.

41. Cragg MS, Kuroda J, Puthalakath H, Huang DC, Strasser A. Gefitinib-induced killing of NSCLC cell lines expressing mutant EGFR requires BIM and can be enhanced by BH3 mimetics. PLoS Med. 2007;4(10):1681-1689.

42. Zhang H, et al. STAT3 restrains RANK- and TLR4-mediated signalling by suppressing expression of the $\mathrm{E} 2$ ubiquitin-conjugating enzyme Ubc13. Nat Commun. 2014;5:5798.

43. Pencik J, et al. STAT3 regulated ARF expression suppresses prostate cancer metastasis. Nat Commun. 2015;6:7736.

44. Peixoto P, et al. HDAC7 inhibition resets STAT3 tumorigenic activity in human glioblastoma independently of EGFR and PTEN: new opportunities for selected targeted therapies. Oncogene. 2016;35(34):4481-4494.

45. Grabner B, et al. Disruption of STAT3 signalling promotes KRAS-induced lung tumorigenesis. Nat Commun. 2015;6:6285.

46. Johnson DE, O'Keefe RA, Grandis JR. Targeting the IL-6/JAK/STAT3 signalling axis in cancer. Nat Rev Clin Oncol. 2018;15(4):234-248.

47. Soria JC, et al. Osimertinib in untreated EGFR-mutated advanced non-small-cell lung cancer. N Engl J Med. 2018;378(2):113-125.

48. Sharma SV, et al. A chromatin-mediated reversible drug-tolerant state in cancer cell subpopulations. Cell. 2010;141(1):69-80.

49. Codony-Servat J, et al. Cancer stem cell biomarkers in EGFR-mutation-positive non-small-cell lung cancer. Clin Lung Cancer. 2019;20(3):167-177.

50. Gold KA, et al. A phase I/II trial combining erlotinib with gamma secretase inhibitor RO4929097 in advanced non-small cell lung cancer (NSCLC). Journal of Clinical Oncology. 2013;31(15_suppl):8104.

51. Fabbrizio E, et al. Negative regulation of transcription by the type II arginine methyltransferase PRMT5. EMBO Rep. 2002;3(7):641-645.

52. Calvayrac $\mathrm{O}$, et al. The RAS-related GTPase RHOB confers resistance to EGFR-tyrosine kinase inhibitors in non-small-cell lung cancer via an AKT-dependent mechanism. EMBO Mol Med. 2017;9(2):238-250. 